\title{
SOME RESULTS FOR BETA FRÉCHET DISTRIBUTION
}

Wagner Barreto-Souzd ${ }^{1 *}$, Gauss M. Cordeird $2{ }^{2}$ and Alexandre B. Sima $\mathfrak{3}^{3+}$

* Departamento de Estatística, Universidade Federal de Pernambuco, Cidade Universitária, 50740-540 - Recife, PE, Brazil

† Departamento de Estatística e Informática, Universidade Federal Rural de Pernambuco, Rua Dom Manoel de Medeiros s/n, 50171-900 - Recife, PE, Brazil

+ Associação Instituto Nacional de Matemática Pura e Aplicada, Estrada Dona Castorina 110, Jardim Botânico, 22460-320 - Rio de Janeiro, RJ, Brazil

\begin{abstract}
Nadarajah and Gupta (2004) introduced the beta Fréchet (BF) distribution, which is a generalization of the exponentiated Fréchet (EF) and Fréchet distributions, and obtained the probability density and cumulative distribution functions. However, they do not investigated its moments and the order statistics. In this paper the BF density function and the density function of the order statistics are expressed as linear combinations of Fréchet density functions. This is important to obtain some mathematical properties of the BF distribution in terms of the corresponding properties of the Fréchet distribution. We derive explicit expansions for the ordinary moments and L-moments and obtain the order statistics and their moments. We also discuss maximum likelihood estimation and calculate the information matrix which was not known. The information matrix is easily numerically determined. Two applications to real data sets are given to illustrate the potentiality of this distribution.
\end{abstract}

Keywords: Beta distribution, Exponentiated Fréchet, Fréchet distribution, Information matrix, Maximum likelihood estimation.

\section{INTRODUCTION}

The Fréchet distribution has applications ranging from accelerated life testing through to earthquakes, floods, horse racing, rainfall, queues in supermarkets, sea currents, wind speeds and track race records. Kotz and Nadarajah (2000) give some applications in their book. In this paper, we discuss the BF distribution which stems from the following idea. Eugene et al. (2002) defined the beta $G$ distribution from a quite arbitrary cumulative distribution

\footnotetext{
${ }^{1}$ E-mail: wagnerbs85@hotmail.com

${ }^{2}$ Corresponding author. E-mail: gausscordeiro@uol.com.br

${ }^{3}$ E-mail: alesimas@impa.br
} 
function (cdf) $G(x)$ by

$$
F(x)=\frac{1}{B(a, b)} \int_{0}^{G(x)} \omega^{a-1}(1-\omega)^{b-1} d \omega,
$$

where $a>0$ and $b>0$ are two additional parameters whose role is to introduce skewness and to vary tail weight and $B(a, b)=\int_{0}^{1} \omega^{a-1}(1-\omega)^{b-1} d \omega$ is the beta function. The class of distributions (11) has an increased attention after the works by Eugene et al. (2002) and Jones (2004). Application of $X=G^{-1}(V)$ to the random variable $V$ following a beta distribution with parameters $a$ and $b, V \sim B(a, b)$ say, yields $X$ with cdf (1).

Eugene et al. (2002) defined the beta normal (BN) distribution by taking $G(x)$ to be the cdf of the normal distribution and derived some of its first moments. General expressions for the moments of the BN distribution were derived (Gupta and Nadarajah, 2004). Nadarajah and Kotz (2004) also introduced the beta Gumbel (BG) distribution by taking $G(x)$ to be the cdf of the Gumbel distribution and provided closed-form expressions for the moments, the asymptotic distribution of the extreme order statistics and discussed the maximum likelihood estimation procedure. Nadarajah and Gupta (2004) introduced the BF distribution by taking $G(x)$ to be the Fréchet distribution, derived the analytical shapes of the probability density function (pdf) and the hazard rate function and calculated the asymptotic distribution of the extreme order statistics. However, they do not investigate expressions for its moments and the information matrix which we do in this paper. Also, Nadarajah and Kotz (2005) worked with the beta exponential (BE) distribution and obtained the moment generating function, the first four cumulants, the asymptotic distribution of the extreme order statistics and discussed the maximum likelihood estimation. We can write (11) as

$$
F(x)=I_{G(x)}(a, b),
$$

where $I_{y}(a, b)=B(a, b)^{-1} \int_{0}^{y} w^{a-1}(1-w)^{b-1} d w$ denotes the incomplete beta function ratio, i.e., the cdf of the beta distribution with parameters $a$ and $b$. For general $a$ and $b$, we can express (2) in terms of the well-known hypergeometric function defined by

$$
{ }_{2} F_{1}(\alpha, \beta, \gamma ; x)=\sum_{i=0}^{\infty} \frac{(\alpha)_{i}(\beta)_{i}}{(\gamma)_{i} i !} x^{i},
$$

where $(\alpha)_{i}=\alpha(\alpha+1) \ldots(\alpha+i-1)$ denotes the ascending factorial. We obtain

$$
F(x)=\frac{G(x)^{a}}{a B(a, b)}{ }_{2} F_{1}(a, 1-b, a+1 ; G(x)) .
$$

The properties of the cdf $F(x)$ for any beta $G$ distribution defined from a parent $G(x)$ in (1), could, in principle, follow from the properties of the hypergeometric function which are well established in the literature; see, for example, Section 9.1 of Gradshteyn and Ryzhik (2000).

The probability density function (pdf) corresponding to (11) can be written in the form

$$
f(x)=\frac{1}{B(a, b)} G(x)^{a-1}\{1-G(x)\}^{b-1} g(x),
$$


where $g(x)=d G(x) / d x$ is the pdf of the parent distribution. The pdf $f(x)$ will be most tractable when the functions $G(x)$ and $g(x)$ have simple analytic expressions as is the case of the Fréchet distribution. Except for some special choices for $G(x)$ in (1), it would appear that the pdf $f(x)$ in (3) will be difficult to deal with.

The cdf and pdf of the Fréchet distribution are, respectively,

$$
G_{\sigma, \lambda}(x)=e^{-\left(\frac{\sigma}{x}\right)^{\lambda}}, \quad x>0
$$

and

$$
g_{\sigma, \lambda}(x)=\lambda \sigma^{\lambda} x^{-(\lambda+1)} e^{-\left(\frac{\sigma}{x}\right)^{\lambda}}, \quad x>0,
$$

where $\sigma>0$ is the scale parameter and $\lambda>0$ is the shape parameter. The $r$ th moment of the Fréchet distribution for $r<\lambda$ is $\mu_{r}^{\prime}=\sigma^{r} \Gamma(1-r / \lambda)$, and then the first four cumulants if $\lambda>4$ are

$$
\begin{gathered}
\kappa_{1}=\sigma g_{1}, \quad \kappa_{2}=\sigma^{2}\left(g_{2}-g_{1}^{2}\right) \\
\kappa_{3}=\frac{g_{3}-3 g_{1} g_{2}+2 g_{1}^{2}}{\left(g_{2}-g_{1}^{2}\right)^{3 / 2}}, \quad \kappa_{4}=\frac{g_{4}-4 g_{1} g_{3}+6 g_{1}^{2} g_{2}-3 g_{1}^{4}}{\left(g_{2}-g_{1}^{2}\right)^{2}}
\end{gathered}
$$

where $g_{k}=\Gamma(1-k / \lambda)$ for $k=1, \ldots, 4$.

Nadarajah and Gupta (2004) give the cdf of the BF distribution with parameters $a>0$, $b>0, \sigma>0$ and $\lambda>0$ (denoted by $\operatorname{BF}(a, b, \sigma, \lambda))$ in the same way from (11) by replacing the parent cdf $G(x)$ by (4)

$$
F(x)=\frac{1}{B(a, b)} \int_{0}^{\exp \left\{-\left(\frac{\sigma}{x}\right)^{\lambda}\right\}} \omega^{a-1}(1-\omega)^{b-1} d \omega=I_{\exp \left\{-\left(\frac{\sigma}{x}\right)^{\lambda}\right\}}(a, b), \quad x>0 .
$$

They also give the corresponding pdf and hazard function, respectively, as

$$
f(x)=\frac{\lambda \sigma^{\lambda}}{B(a, b)} x^{-(\lambda+1)} e^{-a\left(\frac{\sigma}{x}\right)^{\lambda}}\left\{1-e^{-\left(\frac{\sigma}{x}\right)^{\lambda}}\right\}^{b-1}, \quad x>0,
$$

and

$$
\tau(x)=\frac{\lambda \sigma^{\lambda} x^{-(\lambda+1)} e^{-a\left(\frac{\sigma}{x}\right)^{\lambda}}\left\{1-e^{-\left(\frac{\sigma}{x}\right)^{\lambda}}\right\}^{b-1}}{B(a, b)\left\{1-I_{\exp \left\{-\left(\frac{\sigma}{x}\right)^{\lambda}\right\}}(a, b)\right\}}, \quad x>0 .
$$

Figures 1 and 2 illustrate some of the possible shapes of the pdf (6) and hazard function (7), respectively, for selected parameter values, including the case of the Fréchet distribution. The $\mathrm{BF}$ distribution is easily simulated from (5) as follows: if $V \sim B(a, b)$ then $X=\sigma /(-\log V)^{1 / \lambda}$ has the $\operatorname{BF}(a, b, \sigma, \lambda)$ distribution.

The BF distribution generalizes some well-known distributions. The exponentiated Fréchet (EF) distribution (Nadarajah and Kotz, 2003) is a special case when $a=1$. The Fréchet distribution (with parameters $\sigma$ and $\lambda$ ) is also a special case of (6) when $a=b=1$. Further, when $b=1$ and $\lambda=1$, (6) is an inverse gamma distribution with shape parameter 2 and scale parameter $a \sigma$. Since the BF distribution generalizes the Fréchet and EF distributions by adding two parameters and one parameter, respectively, it can be used by practitioners 

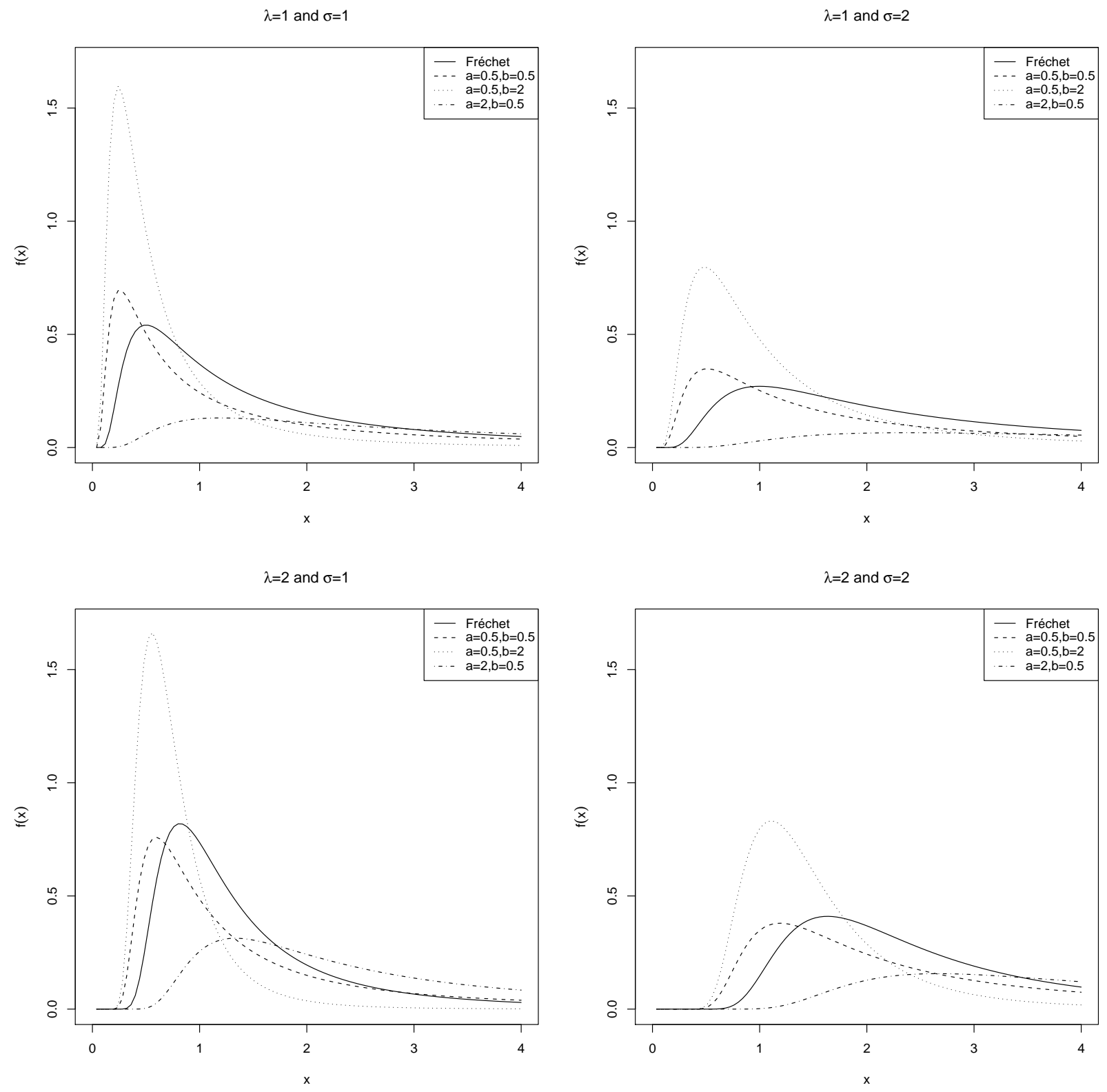

Figure 1: Pdf of the BF distribution for selected parameter values.

as an extra tool to analyze the data we would normally use with the last two distributions. The book of Kotz and Nadarajah (2000) demonstrates the applicability of the Fréchet distribution in several fields.

The rest of the paper is organized as follows. Section 2 gives expansions for the pdf and cdf of the BF distribution and for the density of the order statistics depending on whether the parameters $b$ (or $a>0$ ) is real non-integer or integer. We show that the density functions of the $\mathrm{BF}$ and its order statistics can be expressed as mixture of Fréchet density functions. 

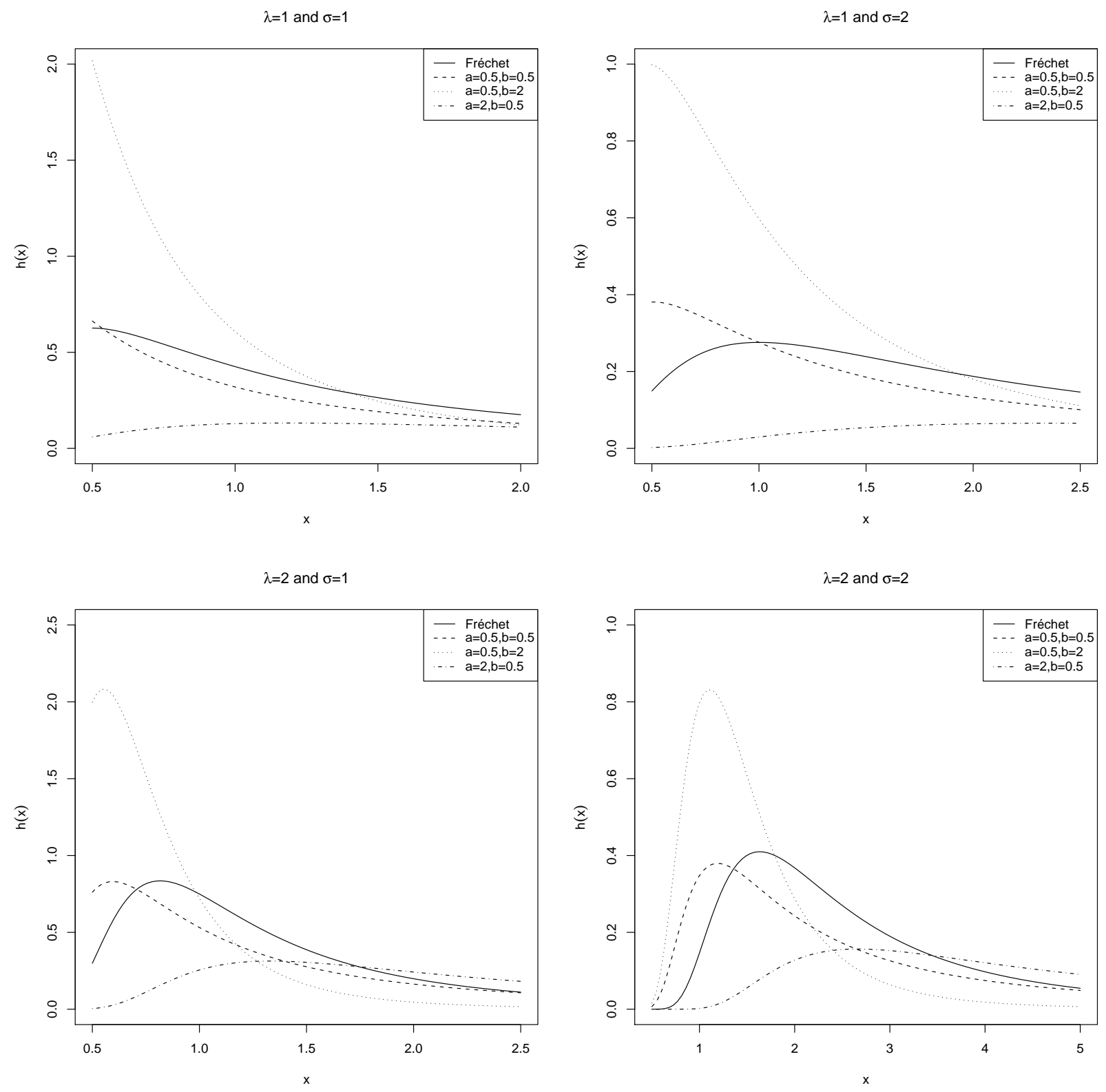

Figure 2: Hazard function of the BF distribution for selected parameter values.

The moments of this distribution and of the order statistics are not known and general expansions are derived in Section 3 for the cases $b>0$ real non-integer and integer. L-moments (Hosking, 1986) are expectations of certain linear combinations of order statistics and form the basis of a general theory which covers the summarization and description of theoretical probability distributions. In Section 4 we present expansions for the L-moments of the BF distribution. We discuss in Section 5 maximum likelihood estimation and calculate the elements of the information matrix. Section 6 provides two applications to real data sets. In 
Section 7 we end with some conclusions.

\section{EXPANSIONS FOR THE DISTRIBUTION AND DENSITY FUNCTIONS}

Here, we provide simple expansions for the cdf of the BF distribution depending on whether the parameter $b$ (or $a$ ) is real non-integer or integer. We consider the series expansion

$$
(1-z)^{b-1}=\sum_{j=0}^{\infty} \frac{(-1)^{j} \Gamma(b)}{\Gamma(b-j) j !} z^{j}
$$

valid for $|z|<1$ and $b>0$ real and non-integer. Application of (8) to (11) if $b$ is real non-integer gives

$$
F(x)=\frac{\Gamma(a+b)}{\Gamma(a)} \sum_{j=0}^{\infty} \frac{(-1)^{j} G_{\sigma, \lambda}(x)^{a+j}}{\Gamma(b-j) j !(a+j)},
$$

where $G_{\sigma, \lambda}(x)$ comes from (44). Then, we have

$$
F(x)=\frac{\Gamma(a+b)}{\Gamma(a)} \sum_{j=0}^{\infty} \frac{(-1)^{j} e^{-(a+j)\left(\frac{\sigma}{x}\right)^{\lambda}}}{\Gamma(b-j) j !(a+j)} .
$$

For $b$ integer, the sum in (10) simply stops at $b-1$. When $b=1$, it follows $F(x)=e^{-a\left(\frac{\sigma}{x}\right)^{\lambda}}$.

It can be seen in the Wolfram Functions Site 4 that for integer $b$

$$
I_{y}(a, b)=\frac{y^{a}}{\Gamma(a)} \sum_{j=0}^{b-1} \frac{\Gamma(a+j)(1-y)^{j}}{j !}
$$

and for integer $a$

$$
I_{y}(a, b)=1-\frac{(1-y)^{b}}{\Gamma(b)} \sum_{j=0}^{a-1} \frac{\Gamma(b+j)}{j !} y^{j} .
$$

Hence, if $b$ is integer, we obtain another equivalent form for (10)

$$
F(x)=\frac{e^{-a\left(\frac{\sigma}{x}\right)^{\lambda}}}{\Gamma(a)} \sum_{j=0}^{b-1} \frac{\Gamma(a+j)\left(1-e^{-\left(\frac{\sigma}{x}\right)^{\lambda}}\right)^{j}}{j !} .
$$

and, for integer values of $a$, we have

$$
F(x)=1-\frac{\left(1-e^{\left.-\left(\frac{\sigma}{x}\right)^{\lambda}\right)^{b}}\right.}{\Gamma(b)} \sum_{j=0}^{a-1} \frac{\Gamma(b+j)}{j !} e^{-j\left(\frac{\sigma}{x}\right)^{\lambda}} .
$$

\footnotetext{
${ }^{4}$ http://functions.wolfram.com/
} 
If $a=1$, the above expression reduces to

$$
F(x)=1-\left\{1-e^{-\left(\frac{\sigma}{x}\right)^{\lambda}}\right\}^{b}
$$

which agrees with the cdf of the EF distribution.

The pdf in (6) is straightforward to compute using any statistical software. However, we show that the BF density can be expressed as an infinite (or finite) weighted linear combination of pdf's of random variables having Fréchet distributions. This is important to provide some mathematical properties of the BF distribution directly from the corresponding properties of the Fréchet distribution. If $b>0$ is real non-integer, and again using (8) we can rewrite (6) as

$$
f(x)=\sum_{k=0}^{\infty} w_{k} g_{a_{k}, \lambda}(x)
$$

where

$$
w_{k}=\Gamma(a+b)(-1)^{k} /\{\Gamma(a) \Gamma(b-k) k !(k+a)\}
$$

represent weighted constants such that $\sum_{k=0}^{\infty} w_{k}=1$ and $g_{a_{k}, \lambda}(x)$ is a Fréchet density with scale parameter $a_{k}=\sigma(k+a)^{1 / \lambda}$ and shape parameter $\lambda$. In addition, if $a=1$, (11) agrees with the corresponding result obtained by Nadarajah and Kotz (2003, Section 5). If $b>0$ is integer, the sum in (11) is finite and stops at $b-1$. Then, the ordinary, central, factorial moments and the moment generating function of the BF distribution could in principle follow from the same weighted infinite (or finite if $b$ is integer) linear combination of the corresponding quantities for the Fréchet distribution.

We now give the density of the $i$ th order statistic $X_{i: n}, f_{i: n}(x)$ say, in a random sample of size $n$ from the BF distribution. It is well known that

$$
f_{i: n}(x)=\frac{1}{B(i, n-i+1)} f(x) F^{i-1}(x)\{1-F(x)\}^{n-i}
$$

for $i=1, \ldots, n$. Using (5) and (6) we can express $f_{i: n}(x)$ in terms of the incomplete beta function ratio

$$
\begin{aligned}
f_{i: n}(x)= & \frac{n ! g_{\sigma, \lambda}(x)}{(i-1) !(n-i) ! B(a, b)} G_{\sigma, \lambda}(x)^{a-1}\left\{1-G_{\sigma, \lambda}(x)\right\}^{b-1} \times \\
& I_{G_{\lambda, \alpha}(x)}(a, b)^{i-1} I_{\left\{1-G_{\lambda, \alpha}(x)\right\}}(b, a)^{n-i} .
\end{aligned}
$$

The cdf of the $i$ th order statistic $X_{i: n}, F_{i: n}(x)$ say, is

$$
F_{i: n}(x)=\sum_{r=i}^{n}\left(\begin{array}{l}
n \\
r
\end{array}\right) I_{G_{\lambda, \alpha}(x)}(a, b)^{r} I_{\left\{1-G_{\lambda, \alpha}(x)\right\}}(b, a)^{n-r} .
$$

Using the identity $\left(\sum_{k=0}^{\infty} a_{k} x^{k}\right)^{n}=\sum_{k=0}^{\infty} c_{k, n} x^{k}$ (see Gradshteyn and Ryzhik, 2000), where $c_{0, n}=a_{0}^{n}$ and

$$
c_{k, n}=\left(k a_{0}\right)^{-1} \sum_{l=1}^{k}(n l-k+l) a_{l} c_{k-l, n}
$$


for $k=1,2, \ldots$ and (10), the pdf of the $i$ th order statistic can be written for $b>0$ real non-integer and integer, respectively, as

$$
f_{i: n}(x)=\sum_{k=0}^{n-i} \sum_{j=0}^{\infty} \frac{(-1)^{k}\left(\begin{array}{c}
n-i \\
k
\end{array}\right) \Gamma(b)^{i+k-1} B(a(i+k)+j, b) c_{i, j, k}^{(1)}}{B(a, b)^{i+k} B(i, n-i+1)} f_{i, j, k}(x)
$$

and

$$
f_{i: n}(x)=\sum_{k=0}^{n-i} \sum_{j=0}^{b-1} \frac{(-1)^{k}\left(\begin{array}{c}
n-i \\
k
\end{array}\right) B(a(i+k)+j, b) c_{i, j, k}^{(2)}}{B(a, b)^{i+k} B(i, n-i+1)} f_{i, j, k}(x),
$$

where $f_{i, j, k}(x)$ is the denstity of a $\mathrm{BF}(a(i+k)+j, b, \sigma, \lambda)$ distribution,

$$
c_{i, 0, k}^{(1)}=\left\{\frac{1}{a \Gamma(b)}\right\}^{i+k-1}, \quad c_{i, j, k}^{(1)}=\frac{a \Gamma(b)}{j} \sum_{l=1}^{j} \frac{(-1)^{l}\{l(i+k)-j\}}{\Gamma(b-l) l !(a+l)} c_{i, j-l, k}
$$

and

$$
c_{i, 0, k}^{(2)}=\left(\frac{1}{a}\right)^{i+k-1}, \quad c_{i, j, k}^{(2)}=\frac{a}{j} \sum_{l=1}^{j} \frac{(-1)^{l}\left(\begin{array}{c}
b-1 \\
l
\end{array}\right)\{l(i+k)-j\}}{a+l} c_{i, j-l, k},
$$

for $j \geq 1$. Expansions (10)-(13) are the main results of this section.

Two alternative expansions for the densities of the order statistics follow from the identity $\left(\sum_{i=1}^{\infty} a_{i}\right)^{k}=\sum_{\left\{m_{1}, \ldots, m_{k}\right\}=0}^{\infty} a_{m_{1}} \ldots a_{m_{k}}$ for $k$ a positive integer. Using this identity and (10), it is easy to show for $b>0$ real non-integer and integer that

$$
f_{i: n}(x)=\sum_{k=0}^{n-i} \sum_{m_{1}=0}^{\infty} \cdots \sum_{m_{i+k-1}=0}^{\infty} \delta_{i, k}^{(1)} f_{i, k}(x)
$$

and

$$
f_{i: n}(x)=\sum_{k=0}^{n-i} \sum_{m_{1}=0}^{b-1} \cdots \sum_{m_{i+k-1}=0}^{b-1} \delta_{i, k}^{(2)} f_{i, k}(x)
$$

respectively, where $f_{i, k}(x)$ is the pdf of a $\operatorname{BF}\left(a(i+k)+\sum_{j=1}^{i+k-1} m_{j}, b, \sigma, \lambda\right)$ distribution,

$$
\delta_{i, k}^{(1)}=\frac{(-1)^{k+\sum_{j=1}^{i+k-1} m_{j}}\left(\begin{array}{c}
n-i \\
k
\end{array}\right) B\left(a(i+k)+\sum_{j=1}^{i+k-1} m_{j}, b\right) \Gamma(b)^{i+k-1}}{B(a, b)^{i+k} B(i, n-i+1) \prod_{j=1}^{i+k-1} \Gamma\left(b-m_{j}\right) m_{j} !\left(a+m_{j}\right)}
$$

and

$$
\delta_{i, k}^{(2)}=\frac{(-1)^{k+\sum_{j=1}^{i+k-1} m_{j}}\left(\begin{array}{c}
n-i \\
k
\end{array}\right) B\left(a(i+k)+\sum_{j=1}^{i+k-1} m_{j}, b\right)}{B(a, b)^{i+k} B(i, n-i+1)} \prod_{j=1}^{k+j-1} \frac{\left(\begin{array}{c}
b-1 \\
m_{j}
\end{array}\right)}{\left(a+m_{j}\right)}
$$


The summation in (14) and (15) extends over all $(i+k)$-tuples $\left(k, m_{1}, \ldots, m_{i+k-1}\right)$ of nonnegative integers and is implementable on a computer. However, expansions (12) and (13) are much simpler to be calculated and their CPU times are usually smaller.

\section{MOMENTS}

As with any other distribution, many of the interesting characteristics and features of the $\mathrm{BF}$ distribution can be studied through the moments. We obtain immediately the $r$ th moment $\mu_{r}^{\prime}$ of the BF distribution from (11) if $r<\lambda$

$$
\mu_{r}^{\prime}=\frac{\sigma^{r} \Gamma(1-r / \lambda) \Gamma(a+b)}{\Gamma(a)} \sum_{j=0}^{\infty} \frac{(-1)^{j}(a+j)^{r / \lambda-1}}{\Gamma(b-j) j !} .
$$

If $b>0$ is integer and $r<\lambda$, the sum stops at $b-1$. If $a=1$ and $r<\lambda$, (16) gives the $r$ th moment of the EF distribution with parameters $b, \sigma$ and $\lambda$ which is a new result for the $\mathrm{EF}$ distribution.

From (12) and (13), we obtain simple expansions for the moments of the order statistics. The $r$ th moment of the $X_{i: n}$ for $b>0$ real non-integer is

$$
E\left(X_{i: n}^{r}\right)=\sum_{k=0}^{n-i} \sum_{j=0}^{\infty} \frac{(-1)^{k}\left(\begin{array}{c}
n-i \\
k
\end{array}\right) \Gamma(b)^{i+k-1} B(a(i+k)+j, b) c_{i, j, k}^{(1)}}{B(a, b)^{i+k} B(i, n-i+1)} E\left(X_{i, j, k}^{r}\right)
$$

and for $b>0$ integer

$$
E\left(X_{i: n}^{r}\right)=\sum_{k=0}^{n-i} \sum_{j=0}^{b-1} \frac{(-1)^{k}\left(\begin{array}{c}
n-i \\
k
\end{array}\right) B(a(i+k)+j, b) c_{i, j, k}^{(2)}}{B(a, b)^{i+k} B(i, n-i+1)} E\left(X_{i, j, k}^{r}\right),
$$

where $X_{i, j, k} \sim \operatorname{BF}(a(i+k)+j, b, \sigma, \lambda)$. From (14) and (15), we obtain two alternative expressions for the moments of the order statistics. These expressions for $b>0$ real noninteger and integer are given by

$$
E\left(X_{i: n}^{r}\right)=\sum_{k=0}^{n-i} \sum_{m_{1}=0}^{\infty} \ldots \sum_{m_{i+k-1}=0}^{\infty} \delta_{i, k}^{(1)} E\left(X_{i, k}^{r}\right)
$$

and

$$
E\left(X_{i: n}^{r}\right)=\sum_{k=0}^{n-i} \sum_{m_{1}=0}^{b-1} \ldots \sum_{m_{i+k-1}=0}^{b-1} \delta_{i, k}^{(2)} E\left(X_{i, k}^{r}\right),
$$

respectively, where $X_{i, k} \sim B F\left(a(i+k)+\sum_{j=1}^{i+k-1} m_{j}, b, \sigma, \lambda\right)$. 

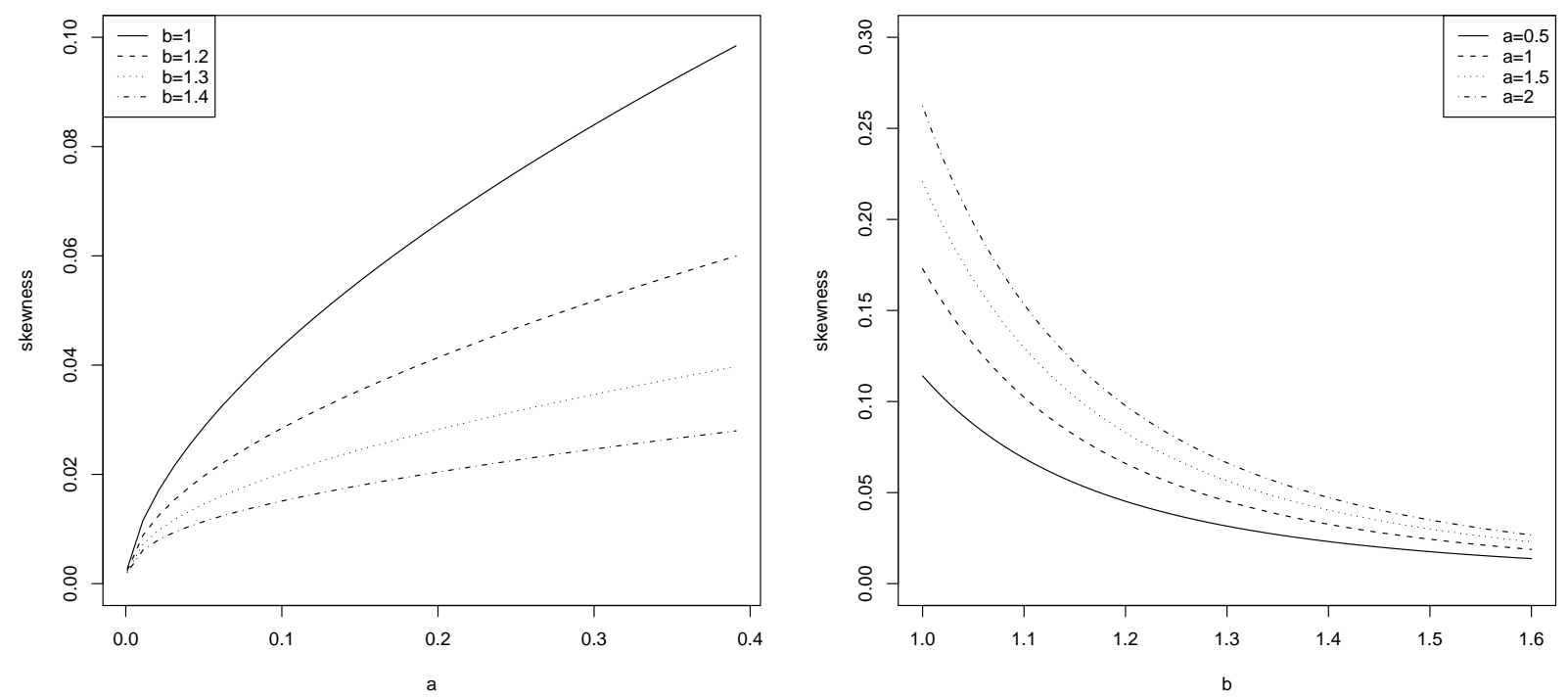

Figure 3: Skewness of the BF distribution as a function of $a(b)$ for selected values of $b(a)$.
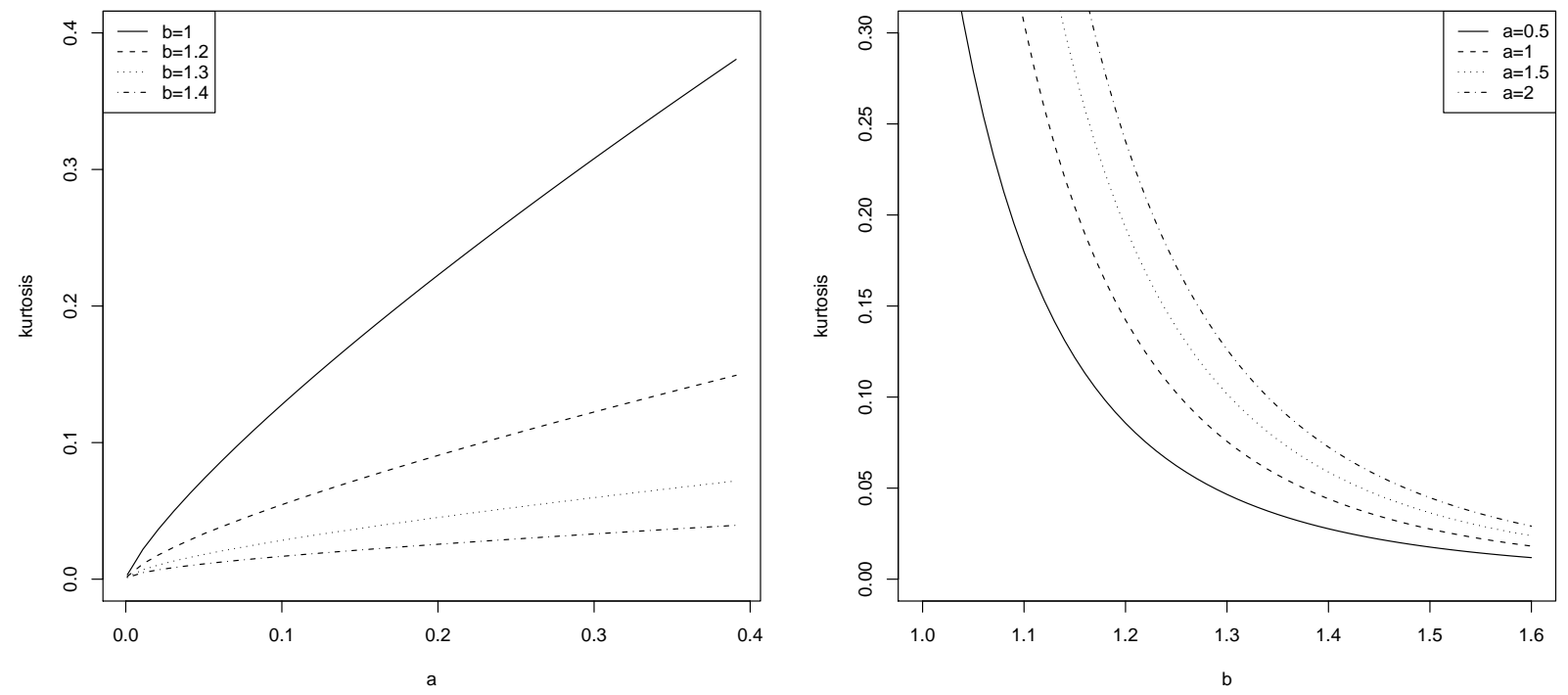

Figure 4: Kurtosis of the BF distribution as a function of $a(b)$ for selected values of $b(a)$.

Graphical representation of skewness and kurtosis when $\lambda=5$ and $\sigma=1$, as a function of parameter $a$ for some choices of parameter $b$, and as a function of parameter $b$ for some choices of parameter $a$, are given in Figures 3 and 4, respectively. 


\section{L-MOMENTS}

The L-moments are analogous to the ordinary moments but can be estimated by linear combinations of order statistics. They are linear functions of expected order statistics defined by (Hosking, 1990)

$$
\lambda_{r+1}=(r+1)^{-1} \sum_{k=0}^{r}(-1)^{k}\left(\begin{array}{l}
r \\
k
\end{array}\right) E\left(X_{r+1-k: r+1}\right), r=0,1, \ldots
$$

The first four L-moments are: $\lambda_{1}=E\left(X_{1: 1}\right), \lambda_{2}=\frac{1}{2} E\left(X_{2: 2}-X_{1: 2}\right), \lambda_{3}=\frac{1}{3} E\left(X_{3: 3}-2 X_{2: 3}+\right.$ $\left.X_{1: 3}\right)$ and $\lambda_{4}=\frac{1}{4} E\left(X_{4: 4}-3 X_{3: 4}+3 X_{2: 4}-X_{1: 4}\right)$. The L-moments have the advantage that they exist whenever the mean of the distribution exists, even though some higher moments may not exist, and are relatively robust to the effects of outliers.

From the expansions (17)-(18) for the moments of the order statistics we can obtain expansions for the L-moments of the BF distribution as weighted linear combinations of the means of suitable BF distributions.

\section{ESTIMATION AND INFORMATION MATRIX}

We assume that $Y$ follows the $\mathrm{BF}$ distribution and let $\theta=(a, b, \sigma, \lambda)^{T}$ be the true parameter vector. The log-likelihood $\ell=\ell(\theta)$ for a single observation $y$ of $Y$ is given by

$$
\ell=\log \lambda+\lambda \log (\sigma / y)-\log \{B(a, b)\}-a(\sigma / y)^{\lambda}+(b-1) \log \left\{1-e^{-(\sigma / y)^{\lambda}}\right\} .
$$

The components of the score vector $U=U(\theta)=(\partial \ell / \partial a, \partial \ell / \partial b, \partial \ell / \partial \sigma, \partial \ell / \partial \lambda)^{T}$ for one observation are given by

$$
\begin{aligned}
\frac{\partial \ell}{\partial a} & =-\psi(a)+\psi(a+b)-(\sigma / y)^{\lambda}, \\
\frac{\partial \ell}{\partial b} & =-\psi(b)+\psi(a+b)+\log \left\{1-e^{-\left(\frac{\sigma}{y}\right)^{\lambda}}\right\}, \\
\frac{\partial \ell}{\partial \sigma} & =\frac{\lambda}{\sigma}-\frac{\lambda \sigma^{\lambda-1}}{y^{\lambda}}\left\{a-\frac{b-1}{e^{\left(\frac{\sigma}{y}\right) \lambda}-1}\right\}, \\
\frac{\partial \ell}{\partial \lambda} & =\frac{1}{\lambda}+\log \left(\frac{\sigma}{y}\right)\left[1-\left(\frac{\sigma}{y}\right)^{\lambda}\left\{a-\frac{b-1}{e^{\left(\frac{\sigma}{y}\right) \lambda}-1}\right\}\right] .
\end{aligned}
$$

From $E(\partial \ell / \partial a)=0$, we obtain

$$
E\left(X^{-\lambda}\right)=\frac{\psi(a+b)-\psi(a)}{\sigma^{\lambda}}
$$

For interval estimation and hypothesis tests on the model parameters, we require the information matrix. The $4 \times 4$ unit information matrix $K=K(\theta)$ is

$$
K=\left(\begin{array}{cccc}
\kappa_{a, a} & \kappa_{a, b} & \kappa_{a, \sigma} & \kappa_{a, \lambda} \\
\kappa_{a, b} & \kappa_{b, b} & \kappa_{b, \sigma} & \kappa_{b, \lambda} \\
\kappa_{a, \sigma} & \kappa_{b, \sigma} & \kappa_{\sigma, \sigma} & \kappa_{\sigma, \lambda} \\
\kappa_{a, \lambda} & \kappa_{b, \lambda} & \kappa_{\sigma, \lambda} & \kappa_{\lambda, \lambda}
\end{array}\right)
$$


whose elements are

$$
\begin{aligned}
\kappa_{a, a}= & \psi^{\prime}(a)-\psi^{\prime}(a+b), \quad \kappa_{b, b}=\psi^{\prime}(b)-\Psi^{\prime}(a+b), \\
\kappa_{\sigma, \sigma}= & \frac{\lambda}{\sigma^{2}}\left[1+a(\lambda-1)\{\psi(a+b)-\psi(a)\}+(b-1)\left(\lambda T_{1,1,2,0}-T_{1,1,1,0}\right)\right], \\
\kappa_{\lambda, \lambda}= & \frac{1}{\lambda^{2}}\left\{1+a T_{0,0,1,2}+(b-1)\left(T_{1,2,2,2}-T_{1,1,1,2}\right)\right\}, \\
\kappa_{\sigma, \lambda}= & -\frac{1}{\sigma}\left[1-a\left\{\psi(a+b)-\psi(a)+T_{0,0,1,1}\right\}+(b-1)\left(T_{1,1,1,0}+T_{1,1,1,1}\right.\right. \\
& \left.\left.-\lambda T_{1,2,2,0}\right)\right], \quad \kappa_{a, b}=-\psi^{\prime}(a+b), \quad \kappa_{a, \lambda}=\frac{1}{\lambda} T_{0,0,1,1}, \\
\kappa_{a, \sigma}= & \frac{\lambda}{\sigma}\{\psi(a+b)-\psi(a)\}, \quad \kappa_{b, \sigma}=-\frac{\lambda}{\sigma} T_{1,1,1,0}, \quad \kappa_{b, \lambda}=-\frac{1}{\lambda} T_{1,1,1,1} .
\end{aligned}
$$

Here, we define a random variable $V$ following a $\operatorname{Bet} a(a, b)$ distribution and the expected value

$$
T_{i, j, k, l}=E\left[V^{i}(1-V)^{-j}(-\log V)^{k}\{\log (-\log V)\}^{l}\right]
$$

where the integral obtained from the above definition is numerically determined using MAPLE and MATHEMATICA for any $a$ and $b$. For example, for $a=1.5$ and $b=2.5$ we easily calculated all $T$ 's in the information matrix: $T_{1,1,2,0}=0.51230070, T_{1,1,1,0}=0.55296103$, $T_{0,0,1,2}=0.62931802, T_{1,2,2,2}=0.43145336, T_{1,1,1,2}=0.32124774, T_{0,0,1,1}=0.48641180$, $T_{1,1,1,1}=-0.16152763$ and $T_{1,2,2,0}=0.86196008$.

For a random sample $y=\left(y_{1}, \ldots, y_{n}\right)^{T}$ of size $n$ from $Y$, the total log-likelihood is

$$
\ell_{n}=\ell_{n}(\theta)=\sum_{i=1}^{n} \ell^{(i)}
$$

where $\ell^{(i)}$ is the log-likelihood for the $i$ th observation $(i=1, \ldots, n)$ as given before. The total score function is $U_{n}=U_{n}(\theta)=\sum_{i=1}^{n} U^{(i)}$, where $U^{(i)}$ for $i=1, \ldots, n$ has the form given earlier and the total information matrix is $K_{n}(\theta)=n K(\theta)$.

The MLE $\hat{\theta}$ of $\theta$ is numerically determined from the solution of the nonlinear system of equations $U_{n}=0$. Under conditions that are fulfilled for the parameter $\theta$ in the interior of the parameter space but not on the boundary, the asymptotic distribution of $\sqrt{n}(\hat{\theta}-$ $\theta)$ is $N_{4}\left(0, K(\theta)^{-1}\right)$. The asymptotic multivariate normal $N_{4}\left(0, K_{n}(\hat{\theta})^{-1}\right)$ distribution of $\hat{\theta}$ can be used to construct approximate confidence regions for some parameters and for the hazard and survival functions. In fact, an $100(1-\gamma) \%$ asymptotic confidence interval for each parameter $\theta_{i}$ is given by

$$
A C I_{i}=\left(\hat{\theta}_{i}-z_{\gamma / 2} \sqrt{\hat{\kappa}^{\theta_{i}, \theta_{i}}}, \hat{\theta}_{i}+z_{\gamma / 2} \sqrt{\hat{\kappa}^{\theta_{i}, \theta_{i}}}\right),
$$

where $\hat{\kappa}^{\theta_{i}, \theta_{i}}$ denotes the $i$ th diagonal element of $K_{n}(\hat{\theta})^{-1}$ for $i=1,2,3,4$ and $z_{\gamma / 2}$ is the quantile $1-\gamma / 2$ of the standard normal distribution. The asymptotic normality is also 
useful for testing goodness of fit of the four parameter BF distribution and for comparing this distribution with some of its special submodels using the likelihood ratio (LR) statistic.

We consider the partition $\theta=\left(\theta_{1}^{T}, \theta_{2}^{T}\right)^{T}$, where $\theta_{1}$ is a subset of the parameters of interest of the $\mathrm{BF}$ and $\theta_{2}$ is a subset of the remaining parameters. The LR statistic for testing the null hypothesis $H_{0}: \theta_{1}=\theta_{1}^{(0)}$ versus the alternative hypothesis $H_{1}: \theta_{1} \neq \theta_{1}^{(0)}$ is given by $w=2\{\ell(\hat{\theta})-\ell(\tilde{\theta})\}$, where $\tilde{\theta}$ and $\hat{\theta}$ denote the MLEs under the null and alternative hypotheses, respectively. The statistic $w$ is asymptotically (as $n \rightarrow \infty)$ distributed as $\chi_{k}^{2}$, where $k$ is the dimension of the subset $\theta_{1}$ of interest. Then, we can compare for example a BF model against an EF model by testing $H_{0}: a=1$ versus $H_{1}: a \neq 1$. We can also compare a $B F$ model against the Fréchet model by testing $H_{0}: a=b=1$ versus $H_{1}: \mathrm{H}_{0}$ is false.

\section{APPLICATIONS}

In this section we fit the $\mathrm{BF}$ distribution to two examples of real data and test two types of hypotheses: $H_{0}$ : Fréchet $\times H_{1}: B F$ and $H_{0}: E F \times H_{1}: B F$. The first example is an uncensored data set from Nichols and Padgett (2006) consisting of 100 observations on breaking stress of carbon fibres (in Gba): 3.7, 2.74, 2.73, 2.5, 3.6, 3.11, 3.27, 2.87, 1.47, 3.11, $4.42,2.41,3.19,3.22,1.69,3.28,3.09,1.87,3.15,4.9,3.75,2.43,2.95,2.97,3.39,2.96,2.53$, $2.67,2.93,3.22,3.39,2.81,4.2,3.33,2.55,3.31,3.31,2.85,2.56,3.56,3.15,2.35,2.55,2.59$, $2.38,2.81,2.77,2.17,2.83,1.92,1.41,3.68,2.97,1.36,0.98,2.76,4.91,3.68,1.84,1.59,3.19$, $1.57,0.81,5.56,1.73,1.59,2,1.22,1.12,1.71,2.17,1.17,5.08,2.48,1.18,3.51,2.17,1.69$, $1.25,4.38,1.84,0.39,3.68,2.48,0.85,1.61,2.79,4.7,2.03,1.8,1.57,1.08,2.03,1.61,2.12$, $1.89,2.88,2.82,2.05,3.65$.

The MLEs and the maximized log-likelihood using the BF distribution are

$$
\hat{a}=0.4108, \quad \hat{b}=125.1891, \quad \hat{\lambda}=0.7496, \quad \hat{\sigma}=31.4556, \quad \hat{\ell}_{B F}=-142.9640,
$$

whereas for the EF and Fréchet distributions we obtain

$$
\hat{b}=52.0491, \quad \hat{\lambda}=0.6181, \quad \hat{\sigma}=26.1730, \quad \hat{\ell}_{E F}=-145.0870,
$$

and

$$
\hat{\lambda}=1.7690, \quad \hat{\sigma}=1.8916, \quad \hat{\ell}_{\text {Fréchet }}=-173.1440,
$$

respectively.

The second data set is obtained from Smith and Naylor (1987). The data are the strengths of $1.5 \mathrm{~cm}$ glass fibres, measured at the National Physical Laboratory, England. Unfortunately, the units of measurement are not given in the paper. The data set is: 0.55, 0.93, $1.25,1.36,1.49,1.52,1.58,1.61,1.64,1.68,1.73,1.81,2,0.74,1.04,1.27,1.39,1.49,1.53$, $1.59,1.61,1.66,1.68,1.76,1.82,2.01,0.77,1.11,1.28,1.42,1.5,1.54,1.6,1.62,1.66,1.69$, $1.76,1.84,2.24,0.81,1.13,1.29,1.48,1.5,1.55,1.61,1.62,1.66,1.7,1.77,1.84,0.84,1.24$, 
$1.3,1.48,1.51,1.55,1.61,1.63,1.67,1.7,1.78,1.89$.

Fitting the BF, EF and Fréchet distributions we obtain the MLEs and the maximized log-likelihood:

$$
\begin{gathered}
\hat{a}=0.3962, \quad \hat{b}=225.7272, \quad \hat{\lambda}=6.8631, \quad \hat{\sigma}=1.3021, \quad \hat{\ell}_{B F}=-90.5180, \\
\hat{b}=112.5986, \quad \hat{\lambda}=7.7859, \quad \hat{\sigma}=0.9814, \quad \hat{\ell}_{E F}=-93.1962
\end{gathered}
$$

and

$$
\hat{\lambda}=1.2643, \quad \hat{\sigma}=2.8875, \quad \hat{\ell}_{\text {Fréchet }}=-117.7765,
$$

respectively.

For the first data set, the values of the LR statistics for testing the hypotheses $H_{0}$ : Fréchet $\times H_{1}: B F$ and $H_{0}: E F \times H_{1}: B F$ are: $60.36\left(\mathrm{p}\right.$-value $\left.=7.81 \times 10^{-14}\right)$ and 4.246 $\left(\mathrm{p}\right.$-value $\left.=3.93 \times 10^{-2}\right)$, respectively. For the second data set, we obtain the values of the LR statistics $54.5170\left(\mathrm{p}\right.$-value $\left.=1.45 \times 10^{-12}\right)$ and $5.3564\left(\mathrm{p}\right.$-value $\left.=2.06 \times 10^{-2}\right)$ for the hypotheses $H_{0}:$ Fréchet $\times H_{1}: B F$ and $H_{0}: E F \times H_{1}: B F$, respectively. Therefore, in both situations, using any usual significance level we reject the null hypotheses in favor of the alternative hypothesis that the BF distribuiton is an adequate model.

The plots of the estimated densities of the BF, EF and Fréchet distributions given in Figure 5 show that the $\mathrm{BF}$ distribution gives a better fit than the other two submodels for both data sets.

\section{CONCLUSIONS}

The BF distribution provides a rather general and flexible framework for statistical analysis of positive data. It unifies some previously proposed distributions, therefore yielding a general overview of these distributions for theoretical studies, and it also provides a rather flexible mechanism for fitting a wide spectrum of real world data sets. The BF distribution is motivated by the wide use of the Fréchet distribution in practice, and also for the fact that the generalization provides more flexibility to analyze more complex situations. In fact, the $\mathrm{BF}$ distribution (6) represents a generalization of some distributions previously considered in the literature such as the Fréchet and EF (Nadarajah and Kotz, 2003) distributions. This generalization provides a continuous crossover towards cases with different shapes (e.g. skewness and kurtosis).

The BF density can be expressed in the mixture form of Fréchet densities. For doing this, we derived some expansions for the cdf of the BF distribution and their ordinary and L-moments. We call attention for the fact that the moments of the EF are not known in the literature and we derived these moments as a particular case of our results. The pdf of the BF order statistics can also be expressed in terms of a linear combination of Fréchet densities. We also derive the moments of the order statistics. We discuss the maximum likelihood 

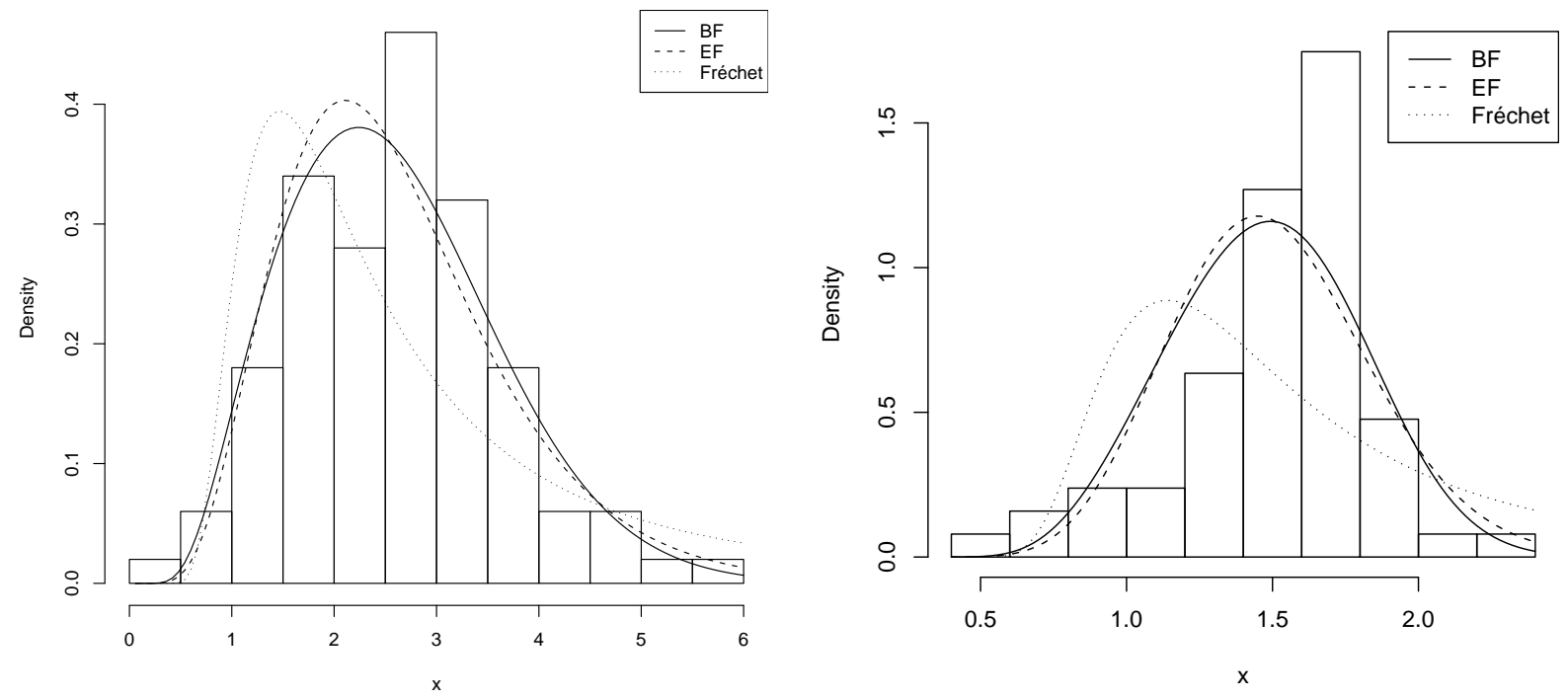

Figure 5: Fitted densities of the BF, EF and Fréchet distributions for the data sets 1 and 2, respectively.

estimation and obtain the information matrix, and considered the LR test which may be very useful in practice. We show that the formulae related with the BF are manageable, and with the use of modern computer resources with analytic and numerical capabilities, may turn into adequate tools comprising the arsenal of applied statisticians. Two numerical examples illustrate that the BF distribution provides better fits than the EF and Fréchet distributions.

\section{BIBLIOGRAPHY}

Birnbaum, Z.W., Saunders, S.C. (1969). Estimation for a family of life distribution with applications to fatigue. Journal of Applied Probability 6:328-347.

Eugene, N., Lee, C., Famoye, F. (2002). Beta-normal distribution and its applications. Commun. Statist. - Theory and Methods 31:497-512.

Gradshteyn, I.S., Ryzhik, I.M. (2000). Table of integrals, series, and products. Academic Press, San Diego.

Gupta, A.K., Nadarajah, S. (2004). On the moments of the beta normal distribution. Commun. Statist. - Theory and Methods 33:1-13.

Hosking, J.R.M. (1990). L-moments: analysis and estimation of distributions using linear 
combinations of order statistics. J. Royal Statist. Soc. B 52:105-124.

Jones, M.C. (2004). Families of distributions arising from distributions of order statistics. Test 13:1-43.

Kotz, S., Nadarajah, S. (2000). Extreme Value Distributions: Theory and Applications. Imperial College Press.

Nadarajah, S., Kotz, S. (2003). The exponentiated Fréchet distribution. InterStat. Available online at http://interstat.statjournals.net/YEAR/2003/abstracts/0312001.php.

Nadarajah, S., Gupta, A.K. (2004). The beta Fréchet distribution. Far East Journal of Theoretical Statistics 14:15-24.

Nadarajah, S., Kotz, S. (2004). The beta Gumbel distribution. Math. Probab. Eng. 10:323332 .

Nadarajah, S. and Kotz, S. (2005). The beta exponential distribution. Reliability Engineering and System Safety 91:689-697.

Smith, R. L. and Naylor, J.C. 1987. A comparison of maximum likelihood and Bayesian estimators for the three-parameter Weibull distribution. Applied Statistics 36: 358-369. 